\title{
Los límites a las políticas del reconocimiento y el derecho a la consulta previa en Honduras El caso garífuna
}

\author{
The Limits of the Politics of Recognition and the Right \\ to Prior Consultation in Honduras \\ The Garifuna Case
}

\author{
Juan Vicente IBORRA MALLENT \\ Universidad Nacional Autónoma de México \\ Maestría en Estudios Latinoamericanos \\ ORCID: 0000-0002-0336-3128 \\ juan.v.iborra@gmail.com
}

\section{Resumen}

En este artículo analizo cómo se han articulado históricamente las políticas de reconocimiento de los grupos indígenas y negros en Honduras a través del análisis del caso garífuna. Mientras que en el periodo neoliberal se implementaron medidas de reconocimiento cultural, los derechos territoriales han sido continuamente asediados por proyectos de desarrollo turístico. Con motivo de la reciente formulación del anteproyecto de Ley de Consulta Previa, Libre e Informada (CPLI) analizo cómo lejos de responder a las demandas de las organizaciones sociales, el derecho a la consulta previa se constituye como un instrumento técnico que contribuye al desplazamiento territorial legitimando la implementación de proyectos de desarrollo económico divergentes de las necesidades de las comunidades locales.

Palabras clave: políticas de reconocimiento, comunidades garífunas, despojo territorial, derecho a la consulta previa.

\begin{abstract}
In this article I analyze the articulation of the politics of recognition of indigenous and black communities in Honduras by focusing the recent Garifun case about minorities' territorial rights. During the neoliberal period in Honduras, various cultural policies were implemented, but territorial rights were continually swindled in the name of tourism development projects. Following the submission of the draft of the Law on Free, Prior and Informed Consultation (CPLI) by the Honduran government to the congress in 2018, I analyze how the "prior consultation" legal resource has been used to displace local communities from their territories to implement instead economic development projects alien to them.
\end{abstract}

Keywords: politics of recognition, Garifuna communities, land grabbing, right to prior consultation. 


\section{Introducción}

La historia de los garífunas ha estado marcada por el despojo y el desplazamiento territorial. En 1797, el Imperio Británico expulsó a los garífunas de la isla de San Vicente, en las Antillas Menores, debido a la oposición de este pueblo a la economía de plantación azucarera. ${ }^{1}$ Posteriormente, los garífunas sufrieron un proceso de dispersión por América Central, conformando núcleos poblacionales en lo que hoy son los países de Belice, Guatemala, Honduras y Nicaragua. A lo largo del siglo xıx ocuparon las franjas territoriales costeras del mar Caribe y se dedicaron al contrabando de mercancías, así como al corte de caoba y otras maderas preciosas. ${ }^{2}$

A finales del siglo xix y principios del $\mathrm{xx}$, con la instalación de las compañías bananeras, los gobiernos iniciaron un proceso de articulación territorial en Honduras que tuvo como consecuencia el progresivo desplazamiento de los garífunas. Los hermanos Vaccaro, fundadores de una compañía que décadas después adoptó el nombre de Standard Fruit Company, reubicaron la comunidad de Armenia al margen opuesto del río Papaloteca. ${ }^{3}$

${ }^{1}$ En plena disputa entre Francia y Gran Bretaña por controlar las Antillas Menores entre los siglos XVII y XVIII, los garífunas fueron denominados caribes negros. La primera distinción entre caribes rojos (de origen caribe y arawako) y caribes negros (de rasgos africanos) se encuentra en los testimonios de Jean-Baptiste Labat (1722). Este imaginario contribuyó a que las autoridades coloniales británicas diferenciaran entre los legítimos pobladores de la isla (caribes rojos o amarillos) y los caribes negros, quienes presentaban condiciones irregulares de vida, carecían de cualquier forma de subordinación establecida y presuntamente cobijaron a cimarrones de otras partes del Caribe. Así, se fue esbozando la expulsión como la solución preferida al "problema caribe". Los hacenderos radicados en Londres llegaron a sugerir el desplazamiento de esta población a "cualquier tramo en la costa de África, de 10000 acres de bosque que estuviera desocupado, y por donde fluyeran uno o más ríos”. Christopher Taylor, The Black Carib Wars. Freedom, Survival and the Making of the Garifuna (Nueva York: St. Martin's Press, 2012), 67. En última instancia, el objetivo era acaparar sus fértiles tierras en un contexto atravesado por el boom azucarero y el agotamiento del suelo en otras islas de la región. Tras múltiples enfrentamientos militares los caribes negros fueron reducidos y trasladados en 1797 primero a la isla de Baliceaux, donde fueron fuertemente diezmados por las epidemias, y más tarde a la isla de Roatán, desde donde se trasladaron al puerto colonial de Trujillo, para posteriormente fundar nuevos núcleos a lo largo de la costa. Sobre la presencia de los caribes en San Vicente véase Nancie L. Solien González, Sojourners of the Caribbean. Ethnogenesis and Ethnohistory of the Garifuna (Urbana: University of Illinois Press, 1988); eds. Peter Hulme y Neil L. Whitehead, Wild Majesty. Encounters with Caribs from Columbus to the Present Day, an Anthology (Oxford: Oxford University Press, 1992).

${ }^{2}$ González, Sojourners of the Caribbean..., 51-73; Antonio Canelas Díaz, El estrangulamiento económico de La Ceiba 1903-1965 (Tegucigalpa: Guaymuras, 2009), 22-23.

${ }^{3}$ Canelas Díaz, El estrangulamiento económico..., 24. 
En La Ceiba, distintos núcleos poblacionales habían sufrido presiones territoriales por parte de finqueros y comerciantes desde finales del siglo XIX. Esta misma situación llevó a que los habitantes de las comunidades de San Antonio, Guadalupe y Punta Hicaco (Santa Fe) solicitaran a la Administración de Rentas de la ciudad de Trujillo un título de propiedad de las tres comunidades, el cual fue concedido en $1885 .{ }^{4}$ Posteriormente, la Comunidad de Morenos Naturales de Cristales y Río Negro obtuvo en 1886 un título de dominio pleno de sus tierras (conocido como el título de La Puntilla), otorgado por Luis Bográn y que fue ampliado a principios del siglo xx por Manuel Bonilla. Al reconocimiento de estas tierras se fueron sumando posteriores ampliaciones. Estas primeras demandas por el reconocimiento de la tierra estuvieron impulsadas por la presión territorial tanto de comerciantes como de las compañías bananeras. En el caso de Trujillo, la familia Melhado participó en la compra y venta de tierras a favor de la Truxillo Railroad Company, subsidiaria de la United Fruit Company en la década de $1930 .^{5}$

En las décadas de 1960 y 1970 la reforma agraria favoreció el proceso de colonización territorial en Honduras, y con ello se extendió la presencia de ganaderos y años después el cultivo masivo de palma africana. ${ }^{6}$ Estos ciclos de acaparamiento de los territorios garífunas se intensificaron durante el periodo neoliberal. El principal mecanismo fue la implantación de grandes proyectos de desarrollo turístico. ${ }^{7} \mathrm{Al}$ mismo tiempo, con el reformismo

${ }^{4}$ Organización Fraternal Negra Hondureña (ofraneh), "Del título del caribal de Punta Hicacos a la Ley de Propiedad”, ofRANeH, acceso el 8 de julio de 2019, http://ofraneh.org/ ofraneh/titulacion.html.

${ }^{5}$ Elizet Payne Iglesias, El puerto de Truxillo, un viaje hacia su melancólico abandono (Tegucigalpa: Guaymuras, 2007), 373.

${ }^{6}$ Respecto a los efectos del reformismo agrario en la costa norte de Honduras se pueden consultar a Mario Mejía, Reforma agraria del Gobierno liberal 1982-1986 y Reforma agraria del gobierno militar 1972-1975. Análisis Comparativo (Tegucigalpa: Universitaria, 1996); Andrés León, "Rebellion Under the Palm Trees, Memory, Agrarian Reform and Labor in the Aguán, Honduras" (Nueva York: CUNY Academic Works, 2015).

${ }^{7}$ Diferentes trabajos han abordado la problemática de despojo territorial a partir del desarrollo turístico en las comunidades garífunas en Honduras. Véanse Keri Vacanti Brondo, Land Grab, Green Neoliberalism, Gender, and Garifuna Resistance in Honduras (Tucson: University of Arizona Press, 2013); Laura Jung, "African Palm and Afro-indigenous Resistance. Race and Dispossession of Garifuna Lands on Honduras' Northern Coast”, n. 84 (Chicago: DePaul University, 2011); Cristopher Anthony Loperena, "Conservation by Racialized Dispossession. The making of an Eco-destination on Honduras's North Coast”, Geoforum, v. 69 (febrero 2016), 184-193, https://doi.org/10.1016/j.geoforum.2015.07.004; Cristopher Anthony Loperena, "Honduras is Open for Business. Extractivist Tourism as Sustainable Development in the Wake of Disaster?", Journal of Sustainable Tourism, v. 25, n. 5 (2017): 618-633, https://doi.org/10.1 
agrario se dio inicio a una nueva coyuntura "etno-racial" ${ }^{8}$ Distintas agencias gubernamentales promovieron la cultura garífuna a través de políticas de reconocimiento cultural.

El objetivo de este artículo es problematizar el vínculo entre el despojo de tierras y el desplazamiento de las comunidades garífunas en Honduras desde una perspectiva histórica. Para ello, realizo una aproximación a los mecanismos que el Estado hondureño ha empleado desde la década de 1960 para enfrentar el problema territorial indígena y garífuna en el país. La hipótesis que planteo, en diálogo con la literatura reciente que cuestiona las políticas de reconocimiento y de construcción de la diferencia, es que durante este periodo y en adelante se crearon condiciones para implementar marcos restrictivos, fundamentados en el reconocimiento limitado de los derechos culturales. Uno de los principales efectos de tales marcos ha recaído en la territorialidad que las organizaciones garífunas reclaman como ancestral. Por tanto, los recientes debates en torno al derecho a la consulta se articulan a los engranajes jurídicos y políticos que históricamente han definido las condiciones de diálogo entre el Estado hondureño y las minorías indígenas y garífunas.

En última instancia, cuestiono cómo la forma de vida garífuna (garifunaduáü) plantea un conflicto ontológico frente al ordenamiento territorial del Estado hondureño. Dicho conflicto se expresa por medio del vínculo entre el territorio y la ritualidad. Su presencia requiere resituar las discusiones en torno al reconocimiento de la diferencia cultural en un contexto atravesado por políticas neoliberales.

De las compañías bananeras al reformismo agrario

La llegada de las compañías bananeras a finales del siglo xix y principios del siglo xx modificó la geografía regional de la Costa Norte de Honduras. En este contexto, la economía de plantación orientada a la exportación

080/09669582.2016.1231808; Tim MacNeill, "Development as Imperialism. Power and the Perpetuation of Poverty in Afro-indigenous Communities of Coastal Honduras", Humanity \& Society, v. 41, n. 2 (2017): 209-239, https://doi.org/10.1177/0160597615603748; Sharlene Mollett, “A Modern Paradise. Garifuna Land, Labor, and Displacement-in-place”, Latin American Perspectives, v. 41, n. 6 (2014): 27-45, https://doi.org/10.1177/0094582X13518756.

${ }^{8}$ Darío Euraque, Conversaciones históricas con el mestizaje y su identidad nacional en Honduras (San Pedro Sula: Centro Editorial, 2004). 
sustituyó a la agricultura de subsistencia. La apropiación de vastas extensiones de tierra y de recursos naturales estratégicos (lagunas, bosques, ríos), las alianzas entre las elites políticas y económicas, ${ }^{9}$ así como el desplazamiento de poblaciones locales para la construcción de puertos e infraestructuras son expresiones de esta transformación.

Simultáneamente, este proceso generó ciclos migratorios de población garífuna a ciudades como Puerto Cortés, San Pedro Sula, Tela o Trujillo. Estos migrantes se incorporaron en la economía bananera como jornaleros agrícolas, auxiliares en puertos o trabajadores de marinos mercantes. ${ }^{10} \mathrm{La}$ huelga bananera de 1954 contribuyó a la legalización de los sindicatos laborales, la ampliación de las prestaciones y la sustitución de formas de empleo estacional por contratos de mayor duración. ${ }^{11}$ Sin embargo, este movimiento también impulsó la mecanización de las formas de producción, reduciendo la demanda de mano de obra y disminuyendo las oportunidades

${ }^{9}$ Esto se dio en distintos niveles de gobierno. Samuel Zemurray tuvo un fuerte apoyo del general Manuel Bonilla y del congreso para obtener amplias franjas de tierra, acceso a recursos hídricos y maderables, a cambio de la construcción de redes ferroviarias. John Soluri, Banana Cultures. Agriculture, Consumption, and Environmental Change in Honduras and the United States (Austin: University of Texas Press, 2005), 476. Zemurray apoyó al gobierno de Bonilla después de su exilio, lo que le permitió obtener mayores concesiones de tierras, exenciones de impuestos e infraestructuras. Darío Euraque, Reinterpreting the Banana Republic. Region and State in Honduras, 1870-1972 (Chapell Hill: University of North Carolina Press, 1996), 7. Tanto la Standard Fruit Company como la United Fruit Company y sus subsidiarias, la Tela Railroad Company y la Truxillo Railroad Company, controlaron decenas de miles de hectáreas. Esto también se observó en el nivel local en lugares como La Ceiba, donde la huelga de trabajadores de 1920 en contra de los hermanos Vaccaro (Standard) fue sofocada por el comandante de armas de la ciudad y gobernador político del departamento de Atlántida, el doctor Antonio Ramón Lagos. Canelas Díaz, El estrangulamiento económico..., 13.

${ }^{10}$ Respecto al papel de los trabajadores garífunas en la economía bananera, véanse Sarah England, Afro Central Americans in New York City. Garifuna Tales of Transnational Movements in Racialized Space (Gainesville: University Press of Florida, 2006); Suyapa G. Portillo Villeda, "Campeñas, campeños y compañeros. Life and Work in the Banana Fincas of the North Coast of Honduras, 1944-1957" (tesis doctoral, Cornell University, 2011). Las compañías bananeras promovieron la llegada de trabajadores extranjeros negros (creoles) procedentes de las Indias Occidentales. Esto contribuyó a la división racial del trabajo y a la conflictividad interétnica. Un trabajo reciente de Chambers reivindica la agencia de estos negros ingreses o creoles más allá de las maniobras que emplearon las compañías para su confrontación con otros grupos de trabajadores a través de prácticas monopolísticas, de control de salarios y de división de los grupos en función de su jerarquía dentro del proceso de producción. Glenn A. Chambers, Race, Nation, and West Indian Immigration to Honduras, 1890-1940 (Baton Rouge: Louisiana State University Press, 2010).

${ }^{11}$ Euraque, Reinterpreting the Banana..., 96. 
laborales. Este hecho marcó el inicio del retorno de muchos trabajadores a sus comunidades de origen y, con ello, la emergencia de nuevas demandas por la ocupación efectiva de tierras que quedaron abandonadas por las compañías bananeras.

Durante este periodo y en un contexto urbano, los garífunas crearon organizaciones para promover la justicia social y los derechos civiles. Entre otras, destacó la Sociedad Abraham Lincoln, fuertemente influida por las luchas de los movimientos negros en Estados Unidos en contra de la discriminación racial. ${ }^{12}$ Algunos garífunas se involucraron en sindicatos de trabajadores y organizaciones estudiantiles. ${ }^{13}$ Paralelamente, la presión territorial marcó nuevas hojas de ruta entre las organizaciones garífunas. Por ejemplo, las demandas sobre la propiedad de la tierra de sectores campesinos impulsó a los garífunas a organizarse frente a la expansión pionera de la colonización agraria promovida por el Instituto Nacional Agrario (INA). El principio de "función social" de la tierra fue fundamental para expropiar las tierras de las comunidades garífunas. ${ }^{14}$ De tal manera que, a través de las municipalidades, las tierras que estuvieron habitadas durante décadas por las comunidades fueron consideradas ociosas o baldías. Así, campesinos sin tierra ocuparon estos territorios y, al mismo tiempo, se promovió su arrendamiento para actividades como la ganadería, la producción agrícola o la extracción de recursos naturales.

Darío Euraque ${ }^{15}$ señala que el periodo posterior a la huelga de 1954 sentó las bases para la democratización de la cultura política del país e

${ }^{12}$ Santos Centeno, Historia del movimiento negro hondureño (Tegucigalpa: Guaymuras, 1997), 84-85.

${ }^{13}$ Centeno, Historia del movimiento..., 106-174; Gloria Marina Lacayo, Desafiando la ignorancia. Biografía del Doctor Alfonso Lacayo Sánchez primer médico garífuna de Honduras (Bloomington: Palibrio, edición Kindle, 2010).

${ }^{14}$ Olivier Cuisset, "Del campo a la ciudad y vice-versa, elementos para la historia del movimiento garífuna en Honduras", Revista de Estudos Jurídicos e Pesquisas sobre as Américas, v. 8, n. 1 (agosto 2014): 9.

15 El historiador hondureño Darío Euraque estudia cómo en la década de 1960 se inició en la Costa Norte un proceso de industrialización periférica a partir de la inversión de capital extranjero y la recepción de créditos al desarrollo manufacturero. Esta coyuntura fortaleció a sectores de la burguesía de San Pedro Sula vinculados a la Cámara de Comercio e Industria de Cortés (CCIC) y a sectores progresistas del Partido Liberal. Entre otros se encontraban Jaime Rosenthal Oliva, Mauricio Weizemblut Oliva, Henry Holst Leiva, Reginaldo Panting, Antonio José Coello Bobadilla y Edmond L. Bográn. Euraque, Reinterpreting the Banana..., 87. En la década de los 90 algunos personajes como Jaime Rosenthal Oliva o Mauricio Weizemblut Oliva se involucraron en el acaparamiento de tierras de las comunidades garífunas de San Juan y Puerto Castilla. 
impidió la polarización social que se instaló en otros países de la región centroamericana. A pesar de ello, la alianza entre las burguesías locales y la inversión extranjera favoreció la entrada de empresarios agroexportadores de palma africana, contribuyendo a la polarización social en el departamento de Colón y especialmente en la región del Bajo Aguán.

En síntesis, la legislación agraria promovió la modernización del sector agrícola a través de la conversión de latifundios y minifundios en empresas comerciales. La meta era redistribuir las tierras privadas y nacionales en desuso entre las empresas asociativas y cooperativas de campesinos, las cuales tendrían acceso a créditos y asistencia técnica. No obstante, estas reformas beneficiaron a sectores agrarios vinculados a la Federación de Agricultores y Ganaderos de Honduras (FEnAGH), dejando de lado la redistribución equitativa de las tierras. ${ }^{16}$ Esta situación derivó en un ciclo de acaparamiento territorial en regiones donde no se contaba con títulos definitivos de propiedad y en donde la titularidad de la tierra era objeto de disputa.

Las políticas de reconocimiento cultural

En las décadas de 1960 y 1970, Honduras vivió un periodo propicio para el reconocimiento de las diferencias culturales de los pueblos indígenas y negros. Por ejemplo, para Euraque, la emergencia de nuevas prácticas culturales de autorreconocimiento se dio en el marco de una "coyuntura etno-racial" promovida con la creación del Instituto Hondureño de Turismo (IHT). ${ }^{17}$ Como señalé antes, este contexto estuvo marcado por la migración garífuna hacia las ciudades y el surgimiento de nuevas organizaciones sociales, lo que abrió espacios de visibilidad y de participación política.

Anteriormente, la población de Honduras había definido a los garífunas como morenos o morenales. ${ }^{18}$ Por su parte y hasta principios del siglo $\mathrm{xx}$,

16 England, Afro Central Americans..., 110-112.

${ }^{17}$ Euraque, Conversaciones históricas..., 212-241.

${ }^{18}$ Como señala Euraque, el maestro Livio Martínez Lalín, originario de Corozal realizó una investigación en torno a la cultura de los “morenos”, como eran llamados los garífunas por familias olanchanas en La Ceiba. Durante la primera mitad del siglo xx predominaron los discursos del mestizaje hondureño, imperando una narrativa indo-hispana que invisibilizaba la negritud hondureña. Euraque, Conversaciones históricas..., 215. 
la literatura antropológica mantuvo la categoría colonial de caribes negros. ${ }^{19}$ Sin embargo, entre 1960 y 1970, se extendió el uso del término garífuna. En este mismo lapso surgieron intelectuales nativos que promovieron la relación de los garífunas de Honduras con África y con San Vicente, contribuyendo a un redescubrimiento de la cultura e historia propias. José Lino Álvarez Sambulá, un joven garífuna originario de San Juan fundó el Cuadro de Danzas Garífunas en 1962 y la Organización Afro Hondureña. De la misma manera, actuó como autoridad en el "Primer Festival de Arte y Cultura Garífuna” organizado en Tegucigalpa y financiado por el IHT. Crisanto Meléndez, también originario de San Juan, co-fundó ambas organizaciones y el Ballet Folklórico Garífuna.

El 15 de mayo de 1972, Meléndez escribió una de las primeras reivindicaciones del origen negro de la cultura garífuna en un artículo publicado en el periódico El Tiempo, titulado "Breve historia del negro en Honduras". Esta publicación coincidió con la celebración del "Primer Gran Carnaval" de la Ceiba. Posteriormente Meléndez publicó sus investigaciones etnomusicológicas en la revista oficial del Ministerio de Cultura, Sectante, la cual utilizó por vez primera el vocablo de "minorías étnicas". ${ }^{20}$ En 1977, Meléndez participó en el Primer Congreso de la Cultura Negra de las Américas organizado en Cali (Colombia). En este congreso coincidió con intelectuales afroamericanos como el antropólogo colombiano Manuel Zapata Olive1la. ${ }^{21}$ De este modo, la reivindicación del origen antillano y africano de los garífunas se alineó con la emergencia de movimientos indígenas y afrodescendientes que, desde diferentes geografías de América Latina, cuestionaron las narrativas del indigenismo oficial.

En un período marcado por el reformismo militar en Honduras, las políticas de reconocimiento étnico vincularon el turismo con la promoción de la cultura, incluyendo a las minorías dentro de los sectores marginales. ${ }^{22}$

${ }^{19}$ Respecto al uso del término en la literatura antropológica, véanse Ruy Galvão de Andrade Coelho, Los negros caribes de Honduras (Tegucigalpa: Guaymuras, 1995); Nancie L. Solien González, La estructura del grupo familiar entre los Caribes-Negros (Ciudad de Guatemala: editorial José de Pineda Ibarra, Ministerio de Educación, 1979); Virginia Kerns, Women and the Ancestors. Black Carib Kinship and Ritual (Chicago: University of Illinois Press, 1997).

${ }^{20}$ Euraque, Conversaciones históricas..., 241-242.

${ }^{21}$ Crisanto Meléndez, Adeija Sisira Gererum Aguburigu Gariganu: el enojo de las sonajas, palabras del ancestro (Tegucigalpa: Graficentro editores).

${ }^{22}$ Euraque, Conversaciones históricas..., 240; Mark Anderson, Black and Indigenous. Garifuna Activism and Consumer Culture in Honduras (Minneapolis: University of Minnesota Press, 2009), 112. 
Esta tendencia estuvo orientada hacia una progresiva disminución de la "dependencia cultural" y hacia el desarrollo de la cultura popular nacional, lo que se impulsó por medio de los planes de desarrollo nacional, las reformas agrarias, el desarrollo forestal y las políticas de industrialización. ${ }^{23}$

Así, el Plan Nacional de Desarrollo Turístico se convirtió en un intento por promover el reconocimiento étnico a través de circuitos culturales y turísticos. La primera expresión de este fenómeno fue el proyecto de desarrollo turístico Tornasal, implementado en las comunidades de Tornabé y Punta Sal, en la Bahía de Tela. El Banco Centroamericano de Integración Económica (вСIE) impulsó este proyecto. Sin embargo, frente a esta situación, en 1972 se constituyeron la Organización Pro Defensa de Tornabé y el patronato de la comunidad para enfrentar el desplazamiento territorial y reclamar la propiedad de las tierras. ${ }^{24}$ Aunque el proyecto estuvo detenido por décadas, fue retomado a inicios de la década de 1990.

En 1976, ante la instalación de la Empresa Nacional Portuaria (ENP), la comunidad de Puerto Castilla padeció un desplazamiento forzado. El general Gustavo Adolfo Álvarez Martínez del cuarto batallón de infantería de La Ceiba obligó a los comuneros de Cristales y Río Negro (Trujillo) a firmar un decreto extrajudicial que condujo al desplazamiento de la comunidad.

Distintas comunidades garífunas, como Limón o Triunfo de la Cruz, fueron víctimas de estos procesos de presión territorial. Como respuesta se dieron nuevos procesos organizativos con el objetivo de responder a las demandas de las comunidades. En 1977, los garífunas conformaron la Organización Fraternal Negra Hondureña (OFRANEH), en cuya creación participaron antiguos miembros de la Sociedad Lincoln. ${ }^{25}$ Desde sus inicios esta organización articuló patronatos, filiales comunitarias e instituciones externas, ${ }^{26}$ promoviendo el vínculo con profesionales e intelectuales establecidos en las ciudades, así como con miembros de las comunidades. Gradualmente, esta situación generó una polarización en el seno de las organizaciones entre los sectores garífunas más proclives a implementar proyectos de desarrollo con el apoyo de instituciones gubernamentales, desde una perspectiva

${ }^{23}$ Euraque, Conversaciones históricas..., 239.

${ }^{24}$ Donaldo Ochoa, Estudio diagnóstico sobre la situación de la tenencia de la tierra de los pueblos indígenas y garífuna (Tegucigalpa: Comisionado Nacional de los Derechos Humanos, 2003), 149.

${ }^{25}$ Euraque, Conversaciones históricas..., 235-246. Sobre la conformación de la Sociedad Lincoln, véase Centeno, Historia del movimiento..., 77-116.

${ }^{26}$ Cuisset, "Del campo a la ciudad...", 95. 
técnica, y los sectores que priorizaban las problemáticas territoriales, en un contexto caracterizado por el acaparamiento de tierras.

Por ejemplo, Hipólito Centeno, coordinador de la OFRANEH, priorizó las problemáticas de las comunidades y promovió el trabajo de base. Con la llegada de Roy Guevara a la coordinación, se incentivaron los proyectos de desarrollo a partir de iniciativas gubernamentales. ${ }^{27}$ Guevara era técnico en planificación, formado en la Secretaría de Planificación, Coordinación y Presupuesto (sEcPlan). A través de esta institución, Guevara organizó en 1987 el "Primer Seminario Taller con los Grupos Étnicos de Honduras". En este foro participaron agencias estatales, representantes étnicos, organizaciones privadas con una orientación indigenista, el IHT y el Consejo de Promoción Indígena de Honduras (соPIH).$^{28}$ El objetivo de este encuentro fue realizar un diagnóstico de los pueblos indígenas (definidos como "etnias autóctonas") que contribuyera a diseñar un proyecto de etnodesarrollo convergente con los lineamientos del Plan Nacional de Desarrollo y con los discursos ambientalistas defendidos por instituciones internacionales como la unesco. Así, la incipiente legislación sobre el patrimonio situó al ecoturismo como una forma de salvaguarda y de rescate ecológico, cultural y étnico, en consonancia con las dinámicas de globalización económica. ${ }^{29}$

En este contexto, los discursos del etnoturismo y etnodesarrollo fungieron como un mecanismo gubernamental para responder a las demandas territoriales de los garífunas. Por tanto, estos nuevos engranajes definieron el terreno de interlocución entre el Estado hondureño y los pueblos indígenas y negros. En 1992 se creó la Confederación de los Pueblos Autóctonos de Honduras (CONPAH) coincidiendo con la celebración continental de los 500 años de Resistencia Indígena, Negra y Popular. La categoría de

${ }^{27}$ En mi tesis de maestría entrevisté a activistas como Horacio Martínez o Celso Guillén que estuvieron involucrados en OFRANEH en dicho período. Algunas de las reflexiones aquí vertidas son vivos reflejos de dichos diálogos. Véase Juan Vicente Iborra Mallent, "Eibuga Hama Wayunagu Garinagu: caminando con los ancestros garífunas. Cosmopolíticas frente al despojo territorial en tiempos de la tercera expulsión" (tesis de maestría en Estudios Latinoamericanos, Universidad Nacional Autónoma de México, 2019).

28 Anderson, Black and Indigenous..., 121.

${ }^{29}$ Esta legislación estuvo impulsada por el artículo 173 de la Constitución de 1982 que rezaba: "El Estado preservará y estimulará las culturas nativas, así como genuinas expresiones del folklore nacional, el arte popular y las artesanías", lo que reflejó una continuidad en el vínculo institucionalizado entre el desarrollo turístico y el reconocimiento cultural. Euraque, Conversaciones históricas..., 252-255. 
"grupos étnicos autóctonos" hizo equivalente la condición compartida de los pueblos indígenas y negros como racialmente diferenciados, desplazados y despojados territorialmente, pobres y con una falta de acceso a recursos básicos. ${ }^{30}$ El reconocimiento de los grupos negros (creoles y garífunas) e indígenas (chortís, tawahkas, pech, tolupanes, misquitos y lencas) como "grupos étnicos autóctonos" supuso la formulación de una categoría para la definición de aquellos sujetos culturalmente distintos. ${ }^{31}$ Además, se implementaron una serie de medidas legales que buscaban extender el acceso de las etnias autóctonas a los programas de educación y justicia, ${ }^{32}$ y se ratificó el convenio 169 de la Organización Internacional del Trabajo (OIT), que entrañaba una ampliación de los derechos territoriales, culturales y lingüísticos, así como de los mecanismos de consulta.

Sin embargo, estas medidas fueron eclipsadas por la aprobación de otras legislaciones que afectaron la propiedad territorial de las comunidades garífunas. En 1990 se aprobó el Decreto 90-90. Éste permitió la adquisición de bienes urbanos en las áreas delimitadas por el artículo 107, el cual prohibía a los extranjeros adquirir tierras a una distancia mayor de 40 kilómetros de la costa y de las fronteras nacionales. Este decreto afectó a propiedades estatales, ejidales y privadas, constituyendo una maniobra legislativa para promover el turismo y facilitar la inversión extranjera. La emisión del decreto contribuyó a la venta de zonas del litoral atlántico y de las Islas de la Bahía habitadas por comunidades garífunas.

De la misma manera, en 1992 se aprobó la Ley para la Modernización y el Desarrollo del Sector Agrícola (LMDA), lo que conllevó a una expansión de la frontera agraria y al aumento del número de tierras privadas. Este proceso marcó la entrada del monocultivo de palma aceitera. Uno de los efectos, en regiones como el Medio y Bajo Aguán, fue la desaparición de cientos de cooperativas de producción agraria fundadas en la década de 1960. Del mismo modo, esto intensificó el proceso de colonización iniciado durante la reforma agraria en las comunidades garífunas. Por último, se aprobó el apartado dedicado a la "propiedad inmueble de los pueblos indígenas y afrodescendientes” incluido en la Ley de Propiedad Decreto Número

${ }^{30}$ Anderson, Black and Indigenous..., 123.

${ }^{31}$ Anderson, Black and Indigenous..., 124.

32 En 1994 se aprobó el Programa Nacional de Educación para las Etnias Autóctonas de Honduras (PRONEEAH) que suponía la puesta en marcha de una educación bilingüe e intercultural. Además se creó la Fiscalía Especial de las Etnias y Patrimonio Cultural que amplió el acceso a la justicia de las minorías étnicas. 
82-2004. Éste contenía cláusulas que protegían los intereses de inversionistas involucrados en casos de acaparamiento de tierras.

La nueva legislación, al favorecer el desarrollo de proyectos turísticos y el monocultivo, maximizó la presión territorial sobre las comunidades garífunas. Como respuesta, estas comunidades iniciaron un fuerte ciclo de movilización con el fin de demandar el reconocimiento de sus títulos colectivos. Estos esfuerzos culminaron en 1996 con la organización de la Marcha por la Justicia y el Desarrollo de los Pueblos, popularmente conocida como "La Marcha de los Tambores". Finalmente, el gobierno accedió a las exigencias de las organizaciones garífunas otorgando títulos definitivos de propiedad así como la legalización de las tierras. En algunos casos este proceso fue progresivo y requirió trámites de ampliación y de rectificación, materializados en sucesivas correcciones. Aun así, los títulos de propiedad sumaron una fracción de tierras muy inferior a la territorialidad ancestral garífuna, es decir, al territorio garífuna habitado históricamente, hecho que fue motivo de rechazo por parte de las organizaciones.

Como respuesta, desde el periodo de Carlos Roberto Reina (19941998), el gobierno de Honduras implementó proyectos de desarrollo integral para las etnias autóctonas como el programa Nuestras Raíces del Fondo Hondureño de Inversión Social (FHIS). Financiados por instituciones como el Banco Mundial -involucradas al mismo tiempo en el desarrollo de proyectos turísticos que atentaron contra los derechos territoriales de las comunidades garífunas, como el Proyecto de la Bahía de Tela- éstos constituyeron un intento de contrarrestar los costos sociales de los programas de ajuste estructural. Además, estas iniciativas eran individuales, contribuyendo a la división interna de las comunidades y creando fracturas entre los beneficiarios y los excluidos. ${ }^{33}$ En resumen, estos programas fueron un intento gubernamental para frenar las movilizaciones y las demandas de los grupos indígenas y negros mediante su participación en políticas públicas.

De este modo, en la década de 1990 se consolidó la tendencia de alinear las políticas culturales con los planes de desarrollo económico. El reconocimiento ambivalente de los derechos étnicos y culturales fue uno de los efectos de este régimen de gobernanza que delimitó y produjo la diferencia

${ }^{33}$ Míriam Miranda y Alfredo López, "El Banco Mundial, el FHIS y la individualización de los proyectos comunitarios”, Alainet (2 de abril de 2008), acceso el 16 de octubre de 2019, https://www.alainet.org/es/active/23240. 
cultural dentro de los márgenes de la economía neoliberal. Esta tendencia ha sido definida por autores como Charles Hale o Mark Anderson como multiculturalismo neoliberal. ${ }^{34}$ En este marco, las medidas promovidas por el gobierno hondureño definieron los espacios de negociación política así como las posibilidades de participación y legitimación en función de los protocolos establecidos. Por tanto, se consolidó una estrategia de diferenciación entre las organizaciones consideradas como interlocutores válidos y ajustados al marco legal vigente, y aquellas que intentaron profundizar y mantener los procesos históricos de defensa de la propiedad colectiva de la tierra.

Esto condujo a una ruptura en los tejidos organizativos garífunas. El 25 de enero de 1992 se creó la Organización de Desarrollo Comunitario (ODEco), con el fin de ampliar la incidencia e inclusión de las comunidades garífunas en los planes de desarrollo del gobierno, particularmente en los proyectos de desarrollo turístico. ${ }^{35}$ Esta organización llegó a ser un interlocutor cercano del gobierno durante los conflictos territoriales.

Con el paso del tiempo, las diferencias entre ODECO y OFRANEH se acentuaron. Un punto de inflexión fue el golpe de estado en 2009. En agosto de 2011 odeco organizó en La Ceiba la primera Cumbre Mundial de Afrodescendientes, en el marco del Año Internacional de los Afrodescendientes, proclamado por Naciones Unidas. Paralelamente, en este periodo se aprobó la Ley de Regiones Especiales para el Desarrollo (RED) para ampliar significativamente la inversión extranjera en el país. ${ }^{36}$ Ante esta coyuntura OFRANEH

${ }^{34}$ La noción de multiculturalismo neoliberal formulada por Charles Hale ha sido recuperada por Mark Anderson y Rony L. Castillo para el caso garífuna. Anderson, Black and Indigenous...; Rony L. Castillo, "Leave or Die, Neoextractivism and the Garifuna Experience in Honduras. Behind the Migrant Caravan, Ethnographic Updates from Central America", Society For Cultural Anthropology (enero 2019), acceso el 15 de octubre de 2019, https:// culanth.org/fieldsights/leave-or-die-neoextractivism-and-the-garifuna-experience-in-honduras. Charles Hale ha analizado la intersección entre las lógicas de gobernanza neoliberal, el reconocimiento parcial de derechos y la creación de "zonas vacías". Charles R. Hale, "Neoliberal Multiculturalism”, PoLAR: Political and Legal Anthropology Review, v. 28, n. 1 (mayo 2005): 10-19. Charles R. Hale, “¿Resistencia para qué? Territory, Autonomy and Neoliberal Entanglements in the 'Empty Spaces' of Central America”, Economy and Society, v. 40, n. 2 (mayo 2011): 184-210, https://doi.org/10.1080/03085147.2011.548947.

35 Cuisset, "Del campo a la ciudad...”, 103.

${ }^{36}$ Las ciudades modelo o Zonas de Empleo y Desarrollo Económico (ZEDE) son espacios de desarrollo económico que incluyen seguridad privada, leyes especiales de regulación laboral y exención de impuestos, con el objetivo de promover la inversión extranjera. Además, estos proyectos han sido acusados de promover el racismo y la expulsión de las poblaciones de sus territorios. OFRANEH, "Honduras, las ciudades modelo (ZEDE) y racismo" (21 de septiem- 
organizó un Foro sobre Acaparamiento de Territorios en África y América Latina. Así, mientras ODECo promovió la participación de las comunidades afrodescendientes en proyectos como el Plan Puebla Panamá o el Corredor Biológico Mesoamericano, validando un modelo predatorio y extractivo, OFRANEH impulsó los procesos de defensa territorial de distintas comunidades garífunas y priorizó la lucha por la autonomía cultural y territorial. ${ }^{37}$ En conclusión, las diferencias entre ambas organizaciones ponen de relieve los límites de las políticas de reconocimiento cultural del Estado hondureño. Dichas políticas están alineadas con proyectos económicos que minan los derechos territoriales garífunas. Como muestro a continuación, al contrastar estas políticas con la forma de vida garífuna (garifunaduáü) es posible visibilizar una diferencia ontológica fundamental.

En el siguiente apartado reflexiono sobre la problemática del derecho a la consulta. Analizo la disputa de este derecho en el marco de las tensiones que implicó la construcción de la diferencia garífuna para el Estado hondureño y los resortes de la política de reconocimiento cultural. Para ello, incorporo en el texto algunas observaciones etnográficas que realicé entre 2018 y 2019, especialmente las relativas a un encuentro comunitario realizado en la colonia Julio Lino en Trujillo en el que participaron decenas de representantes garífunas y en el que se decidió rechazar el anteproyecto de Ley de Consulta Previa, Libre e Informada (CPLI). ${ }^{38}$

El anteproyecto de ley de consulta previa, libre e informada (CPLI)

Pese a que el gobierno hondureño firmó el convenio 169 de la OIT en 1992, no fue hasta 2012 que discutió la posible aprobación de un mecanismo de consulta para los pueblos indígenas. En este contexto las organizaciones indígenas iniciaron procesos de diálogo para proponer un anteproyecto de

bre de 2016), acceso el 13 de julio de 2019, https://ofraneh.wordpress.com/2016/09/21/ honduras-las-ciudades-modelo-zede-y-racismo/.

${ }^{37}$ Anderson, Black and Indigenous..., 165-166.

${ }^{38}$ Dirección de Pueblos Indígenas y Afrohondureños (DINAFroh), "Propuesta anteproyecto de ley marco de consulta y consentimiento previo, libre e informado a los pueblos indígenas y afrohondureños por el estado Honduras", acceso el 12 de julio de 2020, https:// es.scribd.com/document/317721457/Anteproyecto-Ley-de-CPLI-Version-Dinafroh. 
consulta. ${ }^{39}$ Sin embargo, estos esfuerzos fueron desdeñados por el gobierno, que desde entonces ha impulsado la implementación de un anteproyecto de ley apoyado por instituciones como la Dirección de Pueblos Indígenas y Afrohondureños (DINAfroh), la Confederación de Pueblos Autóctonos de Honduras (CONPAH), el Banco Interamericano de Desarrollo (BID) o el Programa de las Naciones Unidas para el Desarrollo (PNUD).

En junio de 2018 cientos de representantes de diferentes comunidades garífunas convocados por OFRANEH se congregaron para discutir el anteproyecto de Ley de Consulta Previa, Libre e Informada (CPLI). ${ }^{40}$ El lugar escogido para este evento fue la colonia Julio Lino: un territorio recuperado en la comunidad de Cristales y Río Negro (Trujillo). Comuneros, miembros de patronatos, comités de defensa de tierras, recuperaciones, juntas de agua y grupos de pescadores se reunieron para discutir un anteproyecto de ley que no contemplaba el derecho a veto y que carecía del consenso de las comunidades indígenas y garífunas afectadas por los proyectos económicos auspiciados por el Estado hondureño.

${ }^{39}$ El Estado hondureño, impulsado por convenios forestales firmados con la Unión Europea en el marco del Proyecto ONU-REDD (Reducción de las emisiones derivadas de la deforestación y degradación de los bosques) planteó la posibilidad de un mecanismo de consulta que de manera formal salvaguardara los derechos humanos. En este contexto el Observatorio de los Derechos Humanos de los Pueblos Indígenas de Honduras (odHPINH) promovió, a través del diputado Rafael Alegría, un anteproyecto de la Ley de Consulta Previa, Libre e Informada que fue rechazado en el congreso. Posteriormente, con el apoyo del Programa de las Naciones Unidas para el Desarrollo (PNUD) se reemprendieron estos esfuerzos, pero sin el apoyo de diferentes organizaciones indígenas. En enero de 2020 el diputado nacionalista Óscar Nájera, quien tiene fuertes intereses en la siembra de palma africana y ha sido señalado por sus vínculos con el narcotráfico, anunció una nueva reglamentación de la ley de consulta, que ha sido cuestionada por distintas organizaciones como OFRANEH y el Consejo Cívico de Organizaciones Populares e Indígenas de Honduras (copinH) como un mecanismo dirigido a favorecer el despojo de los territorios. Véase ofRANEH, "Denunciado al Estado de Honduras por violación al derecho a la consulta de los pueblos indígenas" (13 de octubre de 2011), acceso el 3 julio de 2019, https://ofraneh.wordpress.com/2011/10/13/292/. OFRANEH, "Honduras: El AVA-FLEGT, los REDD y el derecho a la consulta previa de los pueblos indígenas" (6 de junio de 2016), acceso el 4 de agosto de 2019, https://ofraneh.wordpress. com/2016/05/06/honduras-el-ava-flegt-los-redd-y-el-derecho-a-la-consulta-previa-de-lospueblos-indigenas/; OfRANeH, “A 30 años del Convenio 169 de la oit, la destrucción de la consulta previa a manos del Estado" (13 de septiembre de 2019), acceso el 7 de julio de 2019, https://ofraneh.wordpress.com/2019/09/13/30-anos-del-convenio-169-de-la-oit-y-la-destruccion-de-la-consulta-previa-a-manos-del-estado/.

${ }^{40}$ Para la redacción de este apartado he empleado las notas recopiladas durante mi trabajo de campo en junio de 2018 en Honduras. 
Para este mismo año, en los departamentos de Cortés, Atlántida, Colón y Gracias a Dios de la costa caribeña de Honduras se encontraban 46 comunidades garífunas amenazadas por la implementación de megaproyectos de viviendas turísticas y residenciales, programas de desarrollo económico como las "ciudades modelo" o Zonas de Empleo y Desarrollo Económico (ZEDE), acaparamiento de tierras para la ganadería, el narcotráfico, minería y el monocultivo de palma africana, así como la construcción de hidroeléctricas, termoeléctricas, además de puertos turísticos o comerciales.

Como señalé, comunidades desplazadas en el pasado - como Puerto Castilla- son susceptibles, según el artículo 19 del anteproyecto de ley de CPLI, de traslado y reubicación. Además, en el artículo 20 de la misma ley se contemplan restricciones al derecho de la propiedad colectiva de las comunidades. Días antes de la reunión en Trujillo, Malvin Norales, líder de Puerto Castilla y entonces presidente del patronato de la misma comunidad, fue arrestado. Se le acusó de usurpar un lote de tierras comunitarias. En 1996, la ENP cedió este lote a la empresa INTERMARES y posteriormente éste fue reconocido como propiedad de los hermanos Weizemblut Oliva. Desde la presidencia de Rafael Leonardo Callejas (19901994), las operaciones de ventas ilegales en las que se vieron involucrados presidentes de patronatos, testaferros e inversionistas extranjeros fueron en aumento. Estas ventas fueron resultado de la implementación del modelo económico neoliberal en el que el turismo se situó como un eje prioritario de desarrollo en un país que se encontraba en bancarrota y con altos niveles de déficit fiscal.

En este contexto, lejos de constituirse como un mecanismo de decisión y participación de las comunidades afectadas por los proyectos económicos del gobierno, la ley de consulta legalizaba el despojo territorial y de los recursos naturales. Durante décadas, este despojo se ha desarrollado a expensas de los derechos colectivos de las comunidades. La aprobación de la ley viabilizaba el desplazamiento de comunidades como la de Puerto Castilla. Sumado a esto, diversas organizaciones indígenas fueron excluidas de las rondas de consulta, ya que en el desarrollo técnico de esta consulta sólo participaron las organizaciones integradas a la esfera gubernamental (DINAFROH, CONPAH). Una vez más, esto reflejó el intento del Estado hondureño por definir los marcos del diálogo con las organizaciones indígenas.

La reglamentación de la ley de consulta, lejos de esbozar horizontes de reconocimiento de la diferencia cultural y de espacios de autodetermina- 
ción política para los pueblos afectados por las lógicas extractivistas del neoliberalismo actual, da continuidad a las lógicas de desposesión territorial. De este modo, los procesos de desplazamiento histórico encuentran eco en los procesos migratorios recientes. ${ }^{41}$ OFRANEH señaló y denunció este proceso como una tercera expulsión del pueblo garífuna. ${ }^{42}$

Sobre las consultas indígenas se cuenta con una literatura especializada que asocia las lógicas gubernamentales y de legitimación de proyectos extractivistas con el reconocimiento al derecho a la CPLI. Los trabajos de Jessica Argüello Castañón, Raymundo Espinoza y César Rodríguez Garavito documentan cómo los mecanismos de consulta fungen como los entramados burocráticos necesarios para legitimar proyectos de inversión, desarrollo económico e infraestructuras. Por tanto, estos mecanismos no protegen los derechos de las poblaciones indígenas afectadas. ${ }^{43}$

Así, la CPLI participa de un circuito de regímenes jurídicos globales que legitiman políticamente el neoliberalismo. ${ }^{44}$ Por ello, la discusión de la CPLI en Honduras se articuló con las políticas de reconocimiento cultural, lo que explica su carácter ambivalente y contradictorio. En el próximo apartado abordo algunas perspectivas que cuestionan los límites de dichas políticas.

${ }^{41}$ Respecto al actual ciclo migratorio garífuna véase Kenny Castillo Fernández, "Apuntes sobre la migración garífuna en relación a la caravana migrante de hondureños 2018", Diarios del Terruño. Reflexiones sobre Migración y Movilidad, n. 7 (enero-junio 2019): 122-133. Los trabajos que vinculan los ciclos migratorios garífunas a las dinámicas territoriales son escasos, una excepción se puede encontrar en Castillo, "Leave or Die...”.

42 OFRANEH toma como referencia histórica la trata negrera como primera expulsión, y el desplazamiento desde San Vicente como segunda expulsión. Estos dos ciclos constituyen los dos antecedentes del actual y tercer proceso de expulsión, que tiene como síntoma más claro el "vaciamiento" de las comunidades. La proliferación en Centroamérica de zonas vacías, donde la ausencia del estado de derecho implica la reproducción de lógicas económicas predatorias, ha sido enfrentada por las movilizaciones de poblaciones indígenas y negras por la autonomía y la autodeterminación. Hale, “¿Resistencia para qué?...”. Sobre el lugar de las expulsiones en el contexto actual de la economía global véase Saskia Sassen, Expulsiones, brutalidad y complejidad en la economía global (Madrid: Katz editores, 2015).

${ }^{43}$ Véanse César Rodríguez Garavito, Etnicidad.gov, los recursos naturales, los pueblos indígenas y el derecho a la consulta previa en los campos sociales minados (Bogotá: Centro de Estudios de Derecho, Justicia y Sociedad, Dejusticia, 2012); Jessica Argüello Castañón, "La noción de gubernamentalidad de Foucault para analizar los derechos humanos", El Cotidiano, n. 194 (noviembre-diciembre 2015): 79-88; Raymundo Espinoza Hernández, "La consulta a debate, ¿garantía de derechos o mecanismo de despojo?”, Desinformémonos, acceso el 1 de noviembre de 2019, https://desinformemonos.org/wp-content/uploads/2018/11/ La-consulta-a-debate-\%C2\%BFgarant\%C3\%ADa-de-derechos-o-mecanismo-de-despojo_. pdf. Agradezco las sugerencias de Valentina Auletta.

${ }^{44}$ Argüello, "La noción de gubernamentalidad...", 83. 
En este tenor, presento algunos planteamientos teóricos que pueden nutrir la discusión sobre la relación entre el Estado hondureño y la forma de vida garífuna (garifunaduáü).

\section{Autodeterminación más allá del reconocimiento}

Las políticas de reconocimiento emprendidas por el Estado hondureño en las pasadas décadas reflejan ambivalencia y contradicción, ratificando el marcado carácter culturalista de las mismas. Éste es el caso de la promoción de la cultura garífuna por medio del reconocimiento del Ballet Folklórico Garífuna como patrimonio inmaterial de Honduras en 2019. Esta tendencia inició durante el periodo del reformismo agrario y se intensificó con el neoliberalismo. A pesar de que el in a otorgó títulos colectivos sobre la propiedad de las tierras, paralelamente a esta tendencia culturalista, el territorio garífuna ha sufrido ciclos de apropiación y colonización agraria. De igual manera, se ha convertido en un área estratégica para la inversión extranjera interesada en el desarrollo turístico y la promoción de proyectos de viviendas vacacionales y residenciales de lujo.

Más allá de las definiciones estatales de estas tierras, el territorio garífuna es un espacio en el que se realizan rituales ancestrales. En éstos intervienen los espíritus de los parientes fallecidos y suponen la reunión de las familias garífunas, retornando a la comunidad miembros que residen en otras partes del país y en ocasiones en los Estados Unidos y otros países. ${ }^{45}$ A través de rituales como el chugú o el dügü se performan obligaciones con los ancestros: desde la búsqueda de alimentos tradicionales hasta la construcción de un templo en el que se organiza la ceremonia (dabuyaba). Estos rituales son dirigidos por las autoridades espirituales (buyei), las cuales se encargan de mediar entre el mundo de los ancestros y el de los garífunas vivos. Su ejecución requiere meses de preparación y exige la presencia de diferentes parientes, pues tales rituales suponen una reunificación de la familia y la ausencia de algunas personas puede provocar enfermedades o el enfado de los ancestros. Los vínculos rituales de carácter ancestral que se establecen entre los parientes vivos y los

${ }^{45}$ La literatura etnográfica sobre el ritual garífuna es amplia, por lo que no me detendré en presentarla en profundidad. En mi trabajo de maestría abordé el vínculo entre el territorio garífuna y la ritualidad, algunas conclusiones de este escrito nutren las reflexiones vertidas en este apartado. Véase Iborra Mallent, “Eibuga Hama...”, 171-248. 
espíritus de los parientes fallecidos en el contexto del territorio habitado, y que definen la forma de vida garífuna, han sido desdeñados por los marcos normativos existentes. Dichos marcos producidos por el Estado desconocen la diferencia ontológica expresada en la forma de vida garífuna (garifunaduáü). Por el contrario, promueven la definición de las poblaciones garífuna como afrodescendientes, incluyendo a los garífunas dentro de los términos de la ciudadanía hondureña.

Así, a través del multiculturalismo, el Estado hondureño define un marco limitado de derechos culturales. Al divergir de la experiencia garífuna, éste convierte la danza y la música en objetos de folklore que pueden ser integrados en circuitos de consumo globales tales como el turismo. De este modo, se desdeña el vínculo histórico de los garífuna con el territorio ancestral. En éste se sitúa la espiritualidad tradicional, cuya expresión más visible durante los años de relación entre el Estado nación y las comunidades indígenas es la movilización social por las tierras. A pesar de la aparente paradoja en que se insertan las políticas del reconocimiento cultural, los marcos normativos determinan los límites de la participación y de la representación política de los pueblos indígenas y negros en Honduras.

De la misma manera, la conciliación entre las políticas culturales y los grandes proyectos de desarrollo regional - que amenazan con el despojo y desplazamiento de las comunidades garífunas- define los límites del diálogo en el marco del multiculturalismo neoliberal. Por ejemplo, en el imaginario construido de la geografía garífuna, el espacio territorial se conceptualiza como un simple enclave paisajístico con potencial de asimilación en los circuitos de la economía global, negando las formas de vida que subyacen a su realidad material y que expresan una diferencia ontológica que excede las políticas de reconocimiento cultural.

En ese sentido, la construcción histórica de la diferencia garífuna dentro de los marcos interpretativos del multiculturalismo ha insertado la forma de vida de este pueblo (garifunaduáü) en ciertos regímenes de aceptabilidad. La construcción de la diferencia, lejos de contribuir a la constitución de dispositivos de diplomacia cosmopolítica entre diferentes modos de existencia, ${ }^{46}$ ha trazado los límites posibles del diálogo. El encuentro entre distintas formas de vida, en los marcos del pluralismo liberal, está atrave-

${ }^{46}$ Bruno Latour, “¿El cosmos de quién? ¿Qué cosmopolítica? Comentarios sobre los términos de paz de Ulrich Beck”, Pléyade, n. 14 (julio-diciembre 2004): 43-59; Bruno Latour, Investigación sobre los modos de existencia. Una antropología de los modernos (Barcelona: Paidós, 2013). 
sado por la implementación de técnicas biopolíticas de administración de conductas y por la expulsión de poblaciones.

Recientemente, el autor indígena dene, Glen Sean Coulthard, analizó los límites de las políticas de reconocimiento en el marco del pluralismo liberal. ${ }^{47}$ Para Coulthard la desposesión colonial, regida por las lógicas de acumulación, marca el límite inexpugnable y el espacio de traductibilidad del derecho a la diferencia, limitando los espacios de autodeterminación política. Así, las políticas de reconocimiento definen formas de vida naturalizadas que regulan y normativizan las relaciones entre humanos y no humanos. ${ }^{48}$ De tal modo, al alinearse con los procesos de acumulación capitalista, las políticas de reconocimiento estrechan los límites de la discusión política, dejando fuera del ámbito de discusión las formas nativas de obligación y reciprocidad ancladas en la tierra. ${ }^{49}$

En esta misma línea se sitúan los trabajos del antropólogo colombiano Arturo Escobar, quien analiza cómo a través de la reivindicación de derechos territoriales, se manifiesta la existencia de mundos u ontologías relacionales. Así, Escobar considera que el territorio se convierte en un tejido de relacionalidad que asocia de múltiples formas la cultura y la naturaleza a través de conexiones parciales y excesos ontológicos que escapan a las definiciones normativas del pluralismo liberal y de las relaciones capitalistas. ${ }^{50}$

También merece la pena destacar el trabajo de Elizabeth Povinelli, quien analiza los procesos de inclusión asociados con el multiculturalismo liberal australiano y cómo éstos contribuyeron a la difusión de una práctica e ideología de gobernanza que definió los términos de identificación discursiva y representacional. Además, esta antropóloga aborda críticamente el proceso de construcción de las condiciones de posibilidad para realizar reclamaciones de las tierras aborígenes en el marco de conflictos generados por leyes y programas sociales. ${ }^{51}$

${ }^{47}$ Glen Sean Coulthard, Red Skin White Masks. Rejecting the colonial politics of recognition (Minneapolis: University of Minnesota Press, 2014).

${ }^{48}$ Coulthard, Red Skin White Masks..., 140.

${ }^{49}$ Coulthard, Red Skin White Masks..., 13.

${ }^{50}$ Arturo Escobar, "Territorios de diferencia, la ontología política de los 'derechos al territorio”, Cuadernos de Antropología Social, n. 41 (2015): 25-38, https://doi.org/10.34096/ cas.i41.1594.

${ }^{51}$ Elizabeth A. Povinelli, The Cunning of Recognition. Indigenous Alterities and the Making of Australian Multiculturalism (Durham: Duke University Press, 2002); Elizabeth A. Povinelli, Geontologies. A Requiem to Late Liberalism (Durham: Duke University Press, 2016). 
En términos similares se sitúan los planteamientos de autoras como Audra Simpson o Josette Kēhaulani Kauanui. En ambos casos, las autoras discuten sobre cómo los procesos de construcción nacional y de ciudadanía implican la construcción de significados jurídicos en torno a la indigeneidad, la definición de los espacios de soberanía y de reconocimiento, así como la permanencia de estructuras coloniales. ${ }^{52}$

A su vez, considero pertinente ubicar en esta discusión los trabajos del antropólogo Mario Blaser, quien en el contexto del neoliberalismo analiza cómo entre las comunidades yshiro de Paraguay las condiciones de diálogo han estado determinadas por aspectos estructurales y sistémicos. ${ }^{53}$ De este modo, los marcos de negociación se convierten en mecanismos de producción de subjetividad que discriminan entre aquello que es posible y negociable, y aquello que queda fuera de discusión. Así, las lógicas hegemónicas de desarrollo se instituyen en una condición previa al diálogo, más allá de la interlocución entre los actores.

Diferentes propuestas, influidas por el pluralismo jurídico, encontraron en el reconocimiento de los derechos de Sumak Kawsay y Suma Qamaña de las constituciones de Ecuador y Bolivia, otros marcos de inteligibilidad para la inclusión de otras formas de vida en los Estados nación. Así, posturas críticas frente al desarrollo situaron en la emergencia de estos marcos normativos la posibilidad de creación de otros horizontes civilizatorios que incluyeran propuestas de autonomía y pluralismo impulsadas por las movilizaciones indígenas y populares. ${ }^{54}$ Sin embargo, la no transformación en estos países de la matriz productiva agroexportadora ha conducido a conflictos con las comunidades indígenas por la gestión de los recursos naturales, lo que para Maristella Svampa ha supuesto una continuidad del consenso de los commodities que ha contribuido a un fin de ciclo en los gobiernos progresistas ante la caída de los precios internacionales de las

52 Audra Simpson, Mohawk Interruptus. Political Life Across the Borders of Settler States (Durham: Duke University Press, 2014); Josette Kēhaulani Kauanui, Paradoxes of Hawaiian Sovereignty. Land, Sex, and the Colonial Politics of State Nationalism (Durham: Duke University Press, 2018).

${ }^{53}$ Mario Blaser, Un relato de globalización desde el Chaco (Popayán: Universidad del Cauca, 2013).

${ }^{54}$ Eduardo Gudynas y Alberto Acosta, "La renovación de la crítica al desarrollo y el buen vivir como alternativa”, Utopía y Praxis Latinoamericana, v. 16, n. 53 (abril-junio 2011): 71-83. 
materias primas. ${ }^{55}$ Algunos autores como el sociólogo Boaventura de Sousa Santos (2012) observaron, en diálogo con el constitucionalismo crítico y el pluralismo jurídico, la apertura de espacios de reconocimiento de otras epistemologías indígenas en estos emergentes contextos normativos. Un acercamiento interesante fue realizado por el antropólogo Salvador Schavelzon, quien realizó una etnografía del proceso constituyente en Bolivia, abordando cómo en este proceso de yuxtaposición y combinación de la diferencia se autolimitó la soberanía a partir del pluralismo y la autonomía. ${ }^{56}$

Reflexiones finales

En resumen, más que un campo de disputa, el contexto del multiculturalismo en Honduras ha estado atravesado por la gobernanza, la cooptación y el despliegue de limitados marcos de reconocimiento cultural. La construcción de la indigeneidad y de la participación política ha sido condicionada por proyectos hegemónicos de desarrollo económico, lo que explica que el Banco Mundial haya sido uno de los principales impulsores de los mecanismos de consulta.

Por tanto, la reciente CPLI - al instituirse como un mecanismo gubernamental decisorio respecto al legítimo derecho de las comunidades indígenas para decidir sobre problemáticas territoriales que afectan a su forma de vida - suplanta la exigencia de las organizaciones indígenas por dotarse de mecanismos regulatorios y compensatorios que contribuyan a garantizar derechos territoriales y de decisión política. No obstante, la lógica en que se inserta la CPLI remite a un continuum histórico de producción de la diferencia por medio de políticas de reconocimiento cultural, que convierten la forma de vida garífuna (garifunaduáü) en un objeto de folklore, negando el legítimo derecho de las comunidades a decidir sobre sus territorios. Por tanto, el rechazo organizado a la CPLI refleja la existencia de horizontes de autodeterminación ontológica más allá de las políticas de reconocimiento. Así, el vínculo con los ancestros que se establece en el ritual o en la defensa territorial impone un exceso ontológico

55 Maristella Svampa, Del cambio de época al fin de ciclo, gobiernos progresistas, extractivismo, y movimientos sociales en América Latina (Buenos Aires: Edhasa, 2017).

${ }^{56}$ Salvador Schavelzon, "Cosmopolítica y yuxtaposición en la propuesta de Estado Plurinacional de Bolivia”, Revista Chilena de Antropología, n. 33 (enero-junio 2016): 87-101, https://doi.org/10.5354/0719-1472.2016.43391. 
que desborda los limitados marcos estatales de interacción con los pueblos indígenas y negros de Honduras.

\section{BIBLIOGRAFÍA}

Anderson, Mark. Black and Indigenous. Garifuna Activism and Consumer Culture in Honduras. Minneapolis: University of Minnesota Press, 2009.

Andrade Coelho, Ruy Galvão de. Los negros caribes de Honduras. Tegucigalpa: Guaymuras, 1995.

Argüello Castañón, Jessica. "La noción de gubernamentalidad de Foucault para analizar los derechos humanos." El Cotidiano, n. 194 (noviembre-diciembre 2015): 79-88.

Blaser, Mario. Un relato de la Globalización desde el Chaco. Popayán: Universidad del Cauca, 2013.

Brondo, Keri Vacanti. Land Grab. Green Neoliberalism, Gender, and Garifuna Resistance in Honduras. Tucson: University of Arizona Press, 2013.

Canelas Díaz, Antonio. El estrangulamiento económico de La Ceiba 1903-1965. Tegucigalpa: Guaymuras, 2009.

Castillo, Rony L. "Leave or Die. Neoextractivism and the Garifuna Experience in Honduras. Behind the Migrant Caravan: Ethnographic Updates from Central America." Society For Cultural Anthropology (enero 2019), acceso el 15 de octubre de 2019. https://culanth.org/fieldsights/leave-or-die-neoextractivism-and-the-garifuna-experience-in-honduras.

Castillo Fernández, Kenny. "Apuntes sobre la migración garífuna en relación a la caravana migrante de hondureños 2018." Diarios del Terruño. Reflexiones sobre Migración y Movilidad, n. 7 (enero-junio 2019): 122-133.

Centeno, Santos. Historia del movimiento negro hondureño. Tegucigalpa: Guaymuras, 1997.

Chambers, Glenn A. Race, Nation, and West Indian Immigration to Honduras, 18901940. Baton Rouge: Louisiana State University Press, 2010.

Coulthard, Glen Sean. Red Skin White Masks. Rejecting the Colonial Politics of Recognition. Minneapolis: University of Minnesota Press, 2014.

Cuisset, Olivier. "Del campo a la ciudad y vice-versa, elementos para la historia del movimiento garífuna en Honduras." Revista de Estudos Jurídicos e Pesquisas sobre as Américas, v. 8, n. 1 (agosto 2014): 79-111.

Dirección de Pueblos Indígenas y Afrohondureños (DinAfroh), "Propuesta anteproyecto de ley marco de consulta y consentimiento previo, libre e informado 
a los pueblos indígenas y afrohondureños por el Estado Honduras", acceso el 12 de julio 2020. https://es.scribd.com/document/317721457/Anteproyecto-Ley-de-CPLI-Version-Dinafroh.

England, Sarah. Afro Central Americans in New York City. Garifuna Tales of Transnational Movements in Racialized Space. Gainesville: University Press of Florida, 2006.

Escobar, Arturo. "Territorios de diferencia, la ontología política de los 'derechos al territorio'.” Cuadernos de Antropología Social, n. 41 (2015): 25-38. https://doi. org/10.34096/cas.i41.1594.

Espinoza Hernández, Raymundo. "La consulta a debate, ¿garantía de derechos o mecanismo de despojo?" Desinformémonos, acceso el 1 de noviembre de 2019. https://desinformemonos.org/wp-content/uploads/2018/11/La-consulta-a-debate-\%C2\%BFgarant\%C3\%ADa-de-derechos-o-mecanismo-de-despojo_.pdf.

Euraque, Darío. Reinterpreting the Banana Republic. Region and State in Honduras, 1870-1972. Chapell Hill: University of North Carolina Press, 1996.

Euraque, Darío. Conversaciones históricas con el mestizaje y su identidad nacional en Honduras. San Pedro Sula: Centro Editorial, 2004.

González, Nancie L. Solien. La estructura del grupo familiar entre los Caribes-Negros. Ciudad de Guatemala: editorial José de Pineda Ibarra, Ministerio de Educación, 1979.

González, Nancie L. Solien. Sojourners of the Caribbean. Ethnogenesis and Ethnohistory of the Garifuna. Urbana: University of Illinois Press, 1988.

Gudynas, Eduardo y Alberto Acosta. "La renovación de la crítica al desarrollo y el buen vivir como alternativa.” Utopía y Praxis Latinoamericana, v. 16, n. 53 (abril-junio 2011): 71-83.

Hale, Charles R. "Neoliberal multiculturalism." PoLAR: Political and Legal Anthropology Review, v. 28, n. 1 (mayo 2005): 10-19.

Hale, Charles R. “¿Resistencia para qué? Territory, Autonomy and Neoliberal Entanglements in the 'Empty Spaces' of Central America." Economy and Society, v. 40, n. 2 (mayo 2011): 184-210. https://doi.org/10.1080/03085147.2011.548947.

Hulme, Peter y Neil L. Whitehead, eds. Wild Majesty. Encounters With Caribs from Columbus to the Present Day, an Anthology. Oxford: Oxford University Press, 1992. Iborra Mallent, Juan Vicente. “Eibuga Hama Wayunagu Garinagu: caminando con los ancestros garífunas. Cosmopolíticas frente al despojo territorial en tiempos de la tercera expulsión.” Tesis de maestría en Estudios Latinoamericanos. Universidad Nacional Autónoma de México, 2019.

Jung, Laura. "African Palm and Afro-indigenous Resistance. Race and Dispossession of Garifuna lands on Honduras’ Northern Coast”, n. 84. Chicago: DePaul University, 2011. 
Kauanui, Josette Kēhaulani. Paradoxes of Hawaiian Sovereignty. Land, Sex, and the Colonial Politics of State Nationalism. Durham: Duke University Press, 2018.

Kerns, Virginia. Women and the Ancestors. Black Carib Kinship and Ritual. Chicago: University of Illinois Press, 1997.

Lacayo, Gloria Marina. Desafiando la ignorancia. Biografía del doctor Alfonso Lacayo Sánchez, primer médico garífuna de Honduras. Bloomington: Palibrio, edición Kindle, 2010.

Latour, Bruno. “¿El cosmos de quién? ¿Qué cosmopolítica? Comentarios sobre los términos de paz de Ulrich Beck.” Pléyade, n. 14 (julio-diciembre 2004): 43-59.

Latour, Bruno. Investigación sobre los modos de existencia una antropología de los modernos. Barcelona: Paidós, 2013.

León, Andrés. "Rebellion under the Palm Trees. Memory, Agrarian Reform and Labor in the Aguán, Honduras.” Nueva York: Cuny Academic Works, 2015.

Loperena, Cristopher Anthony. "Conservation by Racialized Dispossession. The Making of an Eco-destination on Honduras's North Coast." Geoforum, v. 69 (febrero 2016): 184-193. https://doi.org/10.1016/j.geoforum.2015.07.004.

Loperena, Cristopher Anthony. "Honduras is Open for Business. Extractivist Tourism as Sustainable Development in the Wake of Disaster?" Journal of Sustainable Tourism, v. 25, n. 5 (2017): 618-633. https://doi.org/10.1080/0966958 2.2016.1231808.

MacNeill, Tim. "Development as Imperialism. Power and the Perpetuation of Poverty in Afro-indigenous Communities of Coastal Honduras." Humanity \& Society, v. 41, n. 2 (2017): 209-239. https://doi.org/10.1177/0160597615603748. Mejía, Mario. Reforma agraria del Gobierno liberal 1982-1986 y Reforma Agraria del Gobierno militar 1972-1975 (Análisis Comparativo). Tegucigalpa: Editorial Universitaria, 1996.

Meléndez, Crisanto. Adeija Sisira Gererum Aguburigu Gariganu: el enojo de las sonajas, palabras del ancestro. Tegucigalpa: Graficentro editores.

Miranda, Míriam y Alfredo López. "El Banco Mundial, el FHIS y la individualización de los proyectos comunitarios." Alainet (2 de abril de 2008), acceso el 16 de octubre 2019. https://www.alainet.org/es/active/23240.

Mollett, Sharlene. "A Modern Paradise. Garifuna Land, Labor, and Displacement-in-place.” Latin American Perspectives, v. 41, n. 6 (2014): 27-45. https:// doi.org/10.1177/0094582X13518756.

Ochoa, Donaldo. Estudio diagnóstico sobre la situación de la tenencia de la tierra de los pueblos indígenas y garífuna. Tegucigalpa: Comisionado Nacional de los Derechos Humanos, 2003. 
Organización Fraternal Negra Hondureña (OFRAneH). "Del título del caribal de Punta Hicacos a la Ley de Propiedad.” OFRANEH, acceso el 8 de julio de 2019. http://ofraneh.org/ofraneh/titulacion.html.

OFRANEH. "Denunciado al Estado de Honduras por violación al derecho a la Consulta de los Pueblos Indígenas" (13 de octubre de 2011), OFRANEH, acceso el 3 de julio de 2019. https://ofraneh.wordpress.com/2011/10/13/292/.

OFRANEH. "Honduras, EL AVA-FLEGT, los REDD y el derecho a la consulta previa de los pueblos indígenas" (6 de junio de 2016), OFRANEH, acceso el 4 de agosto de 2019. https://ofraneh.wordpress.com/2016/05/06/honduras-el-ava-flegt-losredd-y-el-derecho-a-la-consulta-previa-de-los-pueblos-indigenas/.

OFRANEH. "Honduras, las ciudades modelo (ZEDE) y racismo" (21 de septiembre de 2016), OFRANEH, acceso el 13 de julio de 2019. https://ofraneh.wordpress. com/2016/09/21/honduras-las-ciudades-modelo-zede-y-racismo/.

OFRANEH. "A 30 años del Convenio 169 de la oIt, la destrucción de la consulta previa a manos del Estado" (13 de septiembre de 2019), OFRANEH, acceso el 7 de julio de 2019. https://ofraneh.wordpress.com/2019/09/13/30-anos-del-convenio-169-de-la-oit-y-la-destruccion-de-la-consulta-previa-a-manos-del-estado/. Payne Iglesias, Elizet. El puerto de Truxillo, un viaje hacia su melancólico abandono. Tegucigalpa: Guaymuras, 2007.

Portillo Villeda, Suyapa G. (2011). “Campeñas, Campeños y Compañeros. Life and Work in the Banana Fincas of the North Coast of Honduras, 1944-1957." Tesis doctoral. Cornell University, 2011.

Povinelli, Elizabeth A. The Cunning of Recognition. Indigenous Alterities and the Making of Australian Multiculturalism. Durham: Duke University Press, 2002.

Povinelli, Elizabeth A. Geontologies. A Requiem to Late Liberalism. Durham: Duke University Press, 2016.

Rodríguez Garavito, César. Etnicidad.gov, los recursos naturales, los pueblos indígenas y el derecho a la consulta previa en los campos sociales minados. Bogotá: Centro de Estudios de Derecho, Justicia y Sociedad, Dejusticia, 2012.

Sassen, Saskia. Expulsiones: brutalidad y complejidad en la economía global. Madrid: Katz editores, 2015.

Schavelzon, Salvador. "Cosmopolítica y yuxtaposición en la propuesta de Estado Plurinacional de Bolivia." Revista Chilena de Antropología, n. 33 (enero-junio 2016): 87-101. https://doi.org/10.5354/0719-1472.2016.43391.

Simpson, Audra. Mohawk Interruptus. Political Life Across the Borders of Settler States. Durham: Duke University Press, 2014.

Soluri, John. Banana Cultures. Agriculture, Consumption, and Environmental Change in Honduras and the United States. Austin: University of Texas Press, 2005. 
Sousa Santos, Boaventura de. "Cuando los excluidos tienen derecho: justicia indígena, plurinacionalidad e interculturalidad." En Justicia indígena, plurinacionalidad e interculturalidad en Ecuador, coord. por Boaventura de Sousa Santos y Agustín Grijalva Jiménez, 13-50. Quito: Abya Yala/Fundación Rosa Luxemburg, 2012.

Svampa, Maristella. Del cambio de época al fin de ciclo, gobiernos progresistas, extractivismo, y movimientos sociales en América Latina. Buenos Aires: Edhasa, 2017. Taylor, Christopher. The Black Carib Wars. Freedom, Survival and the Making of the Garifuna. Nueva York: St. Martin's Press, 2012.

\section{SOBRE EL AUTOR}

Doctorante en Estudios Latinoamericanos en la Universidad Nacional Autónoma de México (UNAM) y becario de investigación Edelberto Torres Rivas en Migraciones y Movilidad Humana en el Consejo Latinoamericano de Ciencias Sociales (Clacso). Ha participado como docente en el Seminario Virtual "Marxismos negros: raza y clase en el pensamiento afrodescendiente y africano" en CLACSO. Entre sus líneas de investigación se encuentran los estudios afrocaribeños y la migración centroamericana. Recientemente ha publicado artículos en las revistas Norteamérica, Tabula Rasa y Humanidades: Revista de la Universidad de Montevideo. 
